\title{
Androgen deprivation therapy and diet: what is the evidence?
}

\author{
Goonewardene $\mathrm{SS}^{1 *}$, Greenwood $\mathrm{R}^{2}$ and Persad $\mathrm{R}^{3}$ \\ ${ }^{1}$ Great Western Hospitals, Swindon, United Kingdom \\ ${ }^{2}$ University of Bristol, United Kingdom \\ ${ }^{3}$ North Bristol NHS Trust, United Kingdom
}

\begin{abstract}
Prostate cancer is the most common cancer in men. With advances in medical therapy, more and more patients are being put on androgen deprivation therapy. Literature demonstrates men undergoing surgery for prostate cancer have significant unmet needs. However, as yet, no systematic review has examined diet and diabetes. Adult males ( $\geq 18,<55$ years) AND Prostate cancer AND Androgen deprivation therapy with dietary supplements, dietary control and diabetes. Intervention: ADT, dietary supplements, diabetes, dietary supplements and lycopene. Experimental vs. Control, Experimental vs. Experimental, Control vs. control. Primary outcomes: Benefit: Improvement in side effects. Harm: Adverse events following treatment. Secondary outcomes: BFRR Suvival outcomes. To assess mens' needs regarding diet and diabetes when on androgen deprivation therapy. A systematic review was conducted of prostate cancer and androgen deprivation therapy, to investigate role of diet and diabetes in androgen deprivation therapy. The systematic review elicited over 580 papers, 10 of which conformed to the search criteria. The dietary requirements were identified from the systematic review by patients as an unmet need. Additionally, very few papers addressed dietary interventions for diabetes when on ADT. These findings were confirmed by existing systematic reviews and conference papers. This systematic review demonstrated a large unmet need for dietary and diabetic care in men on ADT. Poor health related quality of life was also associated with men on ADT, within the systematic review. This study strives to explore dietary and diabetic needs, and subsequent development and implementation of an intervention to address these needs.
\end{abstract}

\section{Introduction}

Patients are experiencing an adverse number of side effects associated with Androgen deprivation therapy (ADT). Whilst there are a number of medical therapies available to combat these side effects, what has not been reviewed are effects of dietary therapy. These include; changes in body composition; an increase in fat mass and a decrease in muscle mass, increased fatigue and a reduced Quality Of Life [1]. Research has demonstrated prognosis may be improved by maintaining healthy weight through diet [2]. Additionally, excess body weight is a known risk factor in high grade prostate cancer [3]. Physiologic mechanisms that are thought to account for these effects of nutritional energy balance on cancer risks include changes in the metabolism of endogenous hormones, growth factors and inflammation factors, as well as in energy and nutrient status at the level of single cells. Physiologic mechanisms including effects of nutritional energy balance on cancer risks include changes in the metabolism of endogenous hormones, growth factors and inflammation factors, as well as in energy and nutrient status [3]. Better knowledge of interaction between diet and the effect of ADT on the body is required. A systematic review was conducted over the past 20 years in order to investigate effect on diet and diabetes on ADT in prostate cancer patients.

\section{Materials and methods}

\section{Subjects and study protocols}

A systematic review relating to literature on dietand diabetes for men with prostate cancer on androgen deprivation therapy was conducted. This was to identify impact of diet or diabetes on or men using androgen deprivation therapy for prostate cancer. The search strategy aimed to identify all references related to prostate cancer AND diet AND androgen deprivation therapy or dietary supplements or complementary medicine or lycopene or diabetes. Search terms used were as follows: (Prostate cancer OR prostate neoplasms) AND (diet) AND (androgen deprivation therapy) OR (dietary supplements) OR (complementary medicine) or (lycopene) or (diabetes).The following databases were screened from 1984 to May 2015: CINAHL, MEDLINE (NHS Evidence), Cochrane, AMed, EMBASE, PsychINFO, SCOPUS, Web of Science.

In addition, searches using Medical Subject Headings (MeSH) and keywords were conducted using Cochrane databases. Two UKbased experts in prostate cancer care were consulted to identify any additional studies.

Studies were eligible for inclusion if they reported primary research focusing on prostate cancer, ADT, diabetes, diet, dietary supplements or complementary medicine or lycopene. Papers were included if published after 1984 and had to be in English. Studies that did not conform to this were excluded. Only primary research was included.

Correspondence to: Sanchia Goonewardene, Great Western Hospitals, Swindon, United Kingdom, E-mail: ssg7727@yahoo.co.uk

Key words: $A D T$, diet, evidence

Received: September 10, 2015; Accepted: October 09, 2015; Published: October 12,2015 
The overall aim was to identify the impact of diet on side effects of ADT in men with prostate cancer.

Abstracts were independently screened for eligibility by two reviewers and disagreements resolved through discussion or third party opinion. Agreement level was calculated using Cohen's Kappa to test the intercoder reliability of this screening process [4]. Cohens' Kappa allows comparison of inter-rater reliability between papers using relative observed agreement. This also takes account of the comparison occurring by chance. The first reviewer agreed all 10 papers to be included, the second, agreed on 36. For this paper, Cohen's kappa was 1.

Figure 1 demonstrates the results of the screening and selection process [5]. Data extraction was piloted by the researcher and amended in consultation with the research team (author and two academic supervisors). Data collected included authors, year and country of publication, study aims, setting, intervention aims, number of participants, study design, intervention components and delivery methods, comparison groups and outcome measures, notes and follow-up questions for the authors. Studies were quality assessed using the PRISMA criteria [5] for randomised controlled trials, Mays et al. [6] for the action research and qualitative studies and the Critical Skills Appraisal programme for cohort studies. This was also applied to randomised controlled trials and qualitative studies. Meta-analyses for the randomised controlled trials was not appropriate as there were only 3 randomised control trials, and the topics being slightly different (with a varying focus of concern). The population used were as follows adult males ( $\geq 18,<55$ years) AND Prostate cancer AND Androgen deprivation therapy with dietary supplements, dietary control and diabetes. Intervention: ADT, dietary supplements, diabetes, dietary supplements and lycopene.For comparisonsExperimental $v s$. Control, Experimental vs. Experimental, Control vs. control were used. For outcomesprimary outcomes used were: Benefit: Improvement in side effects. Harm: Adverse events following treatment. Secondary outcomes used were: biochemical free recurrence rate and survival outcomes.

\section{Results}

The searches identified 580 papers (Figure 1). However, only 10 mapped to the search terms and eligibility criteria. The current systematic reviews were also examined to gain further knowledge about the subject. 580 papers were excluded due to not conforming to eligibility criteria or adding to the evidence for psychosexual pathways or components of pathways for men with prostate cancer, post-surgery. Results were presented as per PRISMA criteria [5] of the 10 papers left, relevant abstracts were identified and the full papers obtained (all of which were in English), to quality assure against criteria. There was significant heterogeneity within studies, including clinical topic, numbers, outcomes, as a result a narrative review was thought to be best.

\section{Characteristics of studies}

\section{Study design was examined (Table 1).}

The randomised controlled trials [7,8] assessed by (Moher et al. 2009) were of the highest quality- 'A' papers'. All studies described withdrawal and dropout rates. They also presented clear and appropriate methods and outcomes. The randomised controlled trials within this review provide good evidence in support of their findings, both towards diet and diabetes having a role in ADT and management of prostate cancer.

For the case control and cohort studies [9-16] they further contributed to understanding of the topic. These papers were of moderate quality [6]. Although these studies were rigorously conducted they all brought out themes of role of diet/ diabetes in ADT and prostate cancer management. All papers gave a clear description of data collection which was appropriately managed. The analysis of each paper was clearly described with adequate discussion. Findings were confirmed in the study, excerpts were transcribed. There was appropriate discussion including an alternative explanation and results of each study are applicable to this area of research. However, no study has sample size calculated, although results were precisely presented.

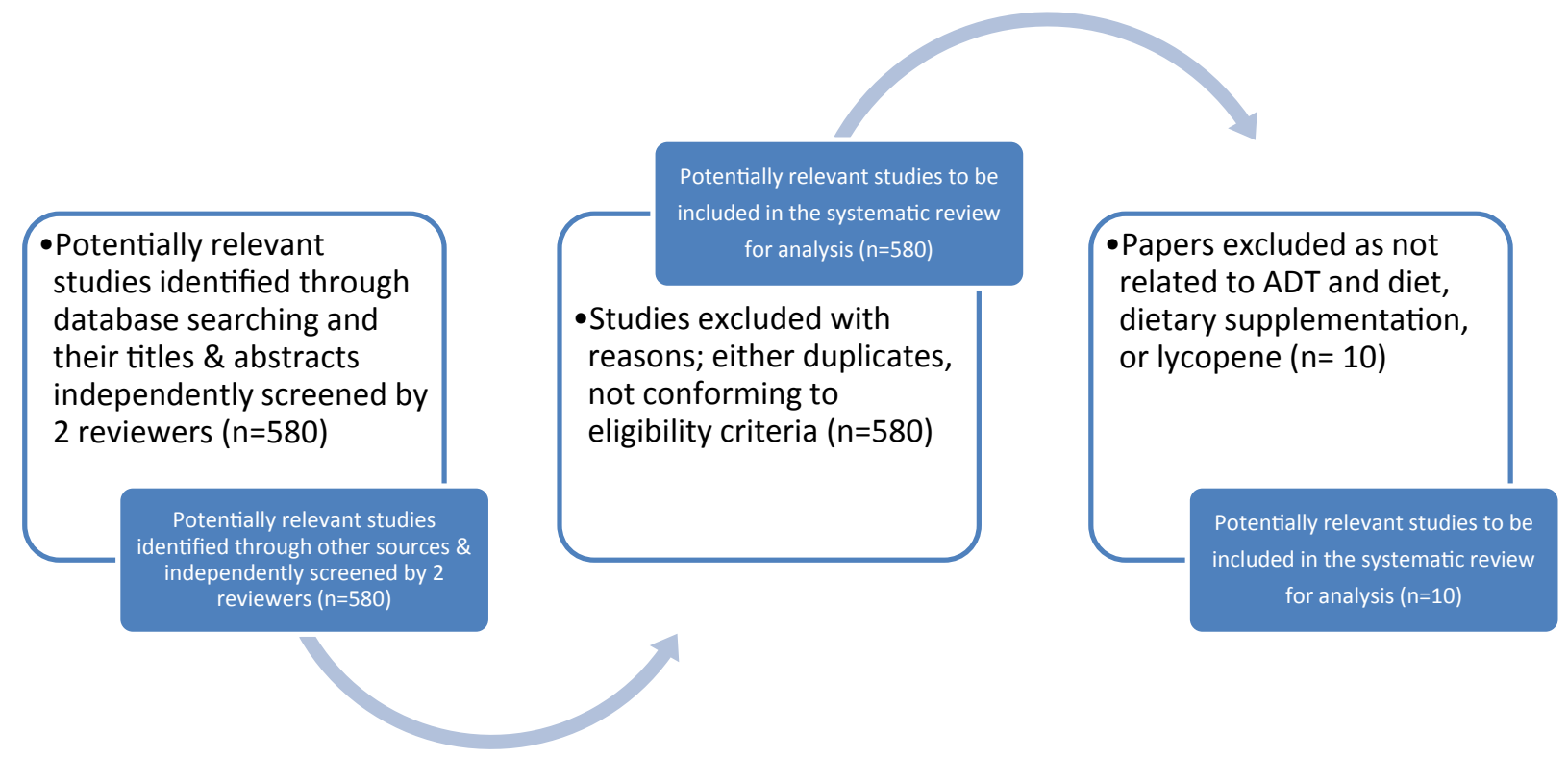

Figure 1. Flow chart of Studies Identified through the Systematic Review (adapted from Moher et al. (2009) PRISMA) [5]. 
Table 1. Types of Study Design in Papers from Systematic Review.

\begin{tabular}{|l|l|}
\hline Type of study design & Papers \\
\hline Randomised controlled trials & (Thomas et al., 2013), (Sharma et al., 2009) [7,8] \\
\hline Case- control study & (Caso et al., 2013) [9] \\
\hline Cohort Study & $\begin{array}{l}\text { (Westerlund et al., 2011) [10] (Keating et al., 2013) [11] (Spratt et al., 2013) [12] (Ziaran } \text { et al., 2013) [13] (Ansari and Gupta, 2004) [14] } \\
\text { (Vaishampayan } \text { et al., 2007) [15] (Lebret et al., 2010) [16] }\end{array}$ \\
\hline
\end{tabular}

Yet, with this kind of research, this will always be a draw back. All studies addressed clearly focused issues relating to diet, diabetes or ADT in prostate cancer. In each paper, the topic was clearly specified and methods were precise. This was presented in a very clear way. Within each of the studies, all study groups were identified in an acceptable way, either from hospital/general practice registries of patients.

Exposures and outcomes were measured, as follows to limit bias [10]. Used a validated food questionnaire to investigate use of dietary supplements in this cohort. Keating et al. [11] used observation of co-morbidities as a cohort study to see impact of this on diabetes and cardiovascular disease for those on ADT [12]. Used PSA as a marker for role of metformin in prostate cancer recurrence. PSA was again used [13-15] to investigate impact of lycopene on ADT therapy in prostate cancer [13]. Used BMI and waist to hip ratio as a measurement of metabolic changes in men [16]. Used a specially developed educational toolkit.All papers, did not have significant loss to follow-up. All results were clearly specified, however and precisely reported.

\section{Categorisation of papers - systematic review} follows:

The papers within this systematic review can be categorised as

\section{Patient education in diet, diabetes and ADT}

Examine patient response to an educational toolkit. After the first visit, $82 \%$ of respondents reported being very glad or glad to receive the kit; among those having read the practical guidance (301/362), $57 \%$ had started implementation and $36 \%$ intended to do so. After the second visit, $76 \%$ were satisfied with the tool-kit and $84 \%$ were implementing guidance. Clinician satisfaction rate was $82 \%$ : benefits were improved patient dialogue (62\%), follow-up (55\%), and better explanation of side effects (51\%). Only 14 clinicians were not pleased by the tool kit. Their main criticisms (too long, tedious, not tailored to individual needs) matched those of patients. Written detailed guidance on diet and physical exercise for patients about to receive ADT meta genuine need and was well perceived by both clinicians and patients. Implementation rate was high. However, content should be adapted to patient age and disease stage [16].

\section{Diabetes and ADT}

Demonstrated, among men with no comorbidities, ADT was associated with an increase of MI. Risks of MI and diabetes was similarly increased among men with and without specific comorbid illnesses [11]. Previous risk factors for MI were associated with new MI and diabetes, and obesity and rheumatologic disease were also associated with diabetes. These demonstrated traditional risk factors for MI and diabetes were also associated with developing these conditions during ADT but did not significantly modify the risk attributable to ADT. This paper also highlighted the important of screening for cardiovascular disease and diabetes in the ADT population. This was confirmed by Lebret et al. [16] who also discovered after 12 months of ADT, BMI, waist to hip ratio, low-density lipoprotein, overall cholesterol increased significantly and (4.2\%) were diagnosed with new onset diabetes. This demonstrated ADT leads into unfavourablechanges in body composition andunfavourable lipoprotein profile.

Spratt et al. determined whether the use of metformin would be associated with improved clinical outcomes and a reduction in the development of castration resistant prostate cancer [12]. Metformin use independently predicted (correcting for PSA, T stage, Gleason score, age, diabetic status, and androgen- deprivation therapy use) improvement in all outcomes compared with the diabetic nonmetformin group. Metformin use was also independently associated with a decrease in the development of CRPC in patients experiencing biochemical failure compared with diabetic non-metformin patients. The retrospective study design was the primary limitation of the study.

\section{Dietary supplements and ADT}

Westerlund et al., [10] examines supplement use in men with prostate cancer and found $48 \%$ had used supplements and had a low intake of saturated fat, compared with population controls.

Ansari and Gupta, [14] examined lycopene treatment in patients with metastatic prostate cancer. (5\%) had complete response (normal PSA, no evidence of disease for 8 weeks). Partial response $(>50 \%$ improvement in PSA level) was achieved in 6 (30\%), disease remained stable in $10(50 \%)$ and progressed in three $(15 \%)$ patients. The conclusions were lycopene therapy improved overall performance status.

This was confirmed by Vaishampayan et al., [15] who also brought in, favourable responses from soy isoflavones. 35 of 37 (95\%) evaluable patients in the lycopene group and 22 of $33(67 \%)$ evaluable patients in the lycopene plus soy isoflavone group achieved stable disease described as stabilization in serum PSA level. The data suggest that lycopene and soy isoflavones have activity in prostate cancer patientswith PSA relapse disease and may delay progression of both hormone-refractory and hormone-sensitive prostate cancer. However, there may not be an additive effect between the 2 compounds when taken together. Future studies are required to investigate this relationship.

\section{Strengths and limitations}

The search criteria of this review included diabetes, diet and androgen deprivation therapy. This approach was focused, robust and the systematic review conducted with rigor. Studies were assessed for both methodological quality and strength of papers. The review is limited by the different methodological studies, requiring narrative review and weak papers, with often wrong methodology or incorrectly matched. It was a relatively heterogeneous population, indicating the 
conclusions published are valid. The lack of papers shows how much more needs to be developed and implemented within this field on a research basis.

However, due to a lack of research in this area, the author feels there were very few papers in this area, that were particularly weak with major gaps in methodology. In addition, as only published studies were included, some relevant ongoing studies may have been excluded- to resolve this, we must examine conferences papers and 'grey literature.' This again will impact on our overall conclusions.

\section{Discussion}

ADT is also associated with worsening diabetic control, cardiovascular disease, and stroke. Lifestyle changes and unmet patient needs, including exercise therapy, diet, and nutrition, have been highlighted time and time again in the literature [17].

In men with prostate cancer [18] GnRH agonists are associated with greater risk of diabetes mellitus and cardiovascular disease. Treatmentrelated obesity and insulin resistance appear sufficient to explain the greater risk for diabetes. Several mechanisms may contribute to greater risk for cardiovascular disease including obesity, insulin resistance, and increased serum cholesterol and triglycerides

Previously, Mostaghel et al. [19] found a critical role for cholesterol in prostate cancer progression to advanced disease by providing evidence that hypercholesterolemia may increase intratumoral de novo steroidogenesis. Originally thought to be hormone refractive, it is now known that CRPC is responsive to further reduction of intracellular androgen levels. Treatment with abiraterone, an inhibitor of androgen biosynthesis in the noncastrate state, highercholesterol may drive tumor. It is also now well established that patients with T2D are at a greater risk for developing numerous cancers, although the exact mechanistic link is still under intense investigation [20]. Given this relationship, it is not surprising that concerns have surfaced regarding the potential effect of antidiabetic agents.

Trials of weight loss interventions in prostate cancerpatients are needed as well as trials to reduce the substantial comorbidity and increased QOL [2]. Some possible ways to be considered to enhance participation include encouragement of "supportive others" such as an oncologist and incorporating home-based exercise and behavioural change techniques [21-23].

The metabolic alterations associated with GnRH agonist therapy appear distinct from the classically defined metabolic syndrome. Future research should focus on betterunderstanding the metabolic consequences of GnRH agonist therapy and developing effective strategies to reduce treatment-related morbidity $[24,25]$.

\section{Conclusions}

In conclusion, roles for management of diabetic disease, and dietary supplements have a path in androgen deprivation therapy management of prostate cancer.

\section{References}

1. Oneill R, Murray L, O'Sullivan J, Haseen F, Cantwell MM (2012) A randomised controlled trial to evaluate the efficacy of a dietary and physical activity intervention on prostate cancer patients receiving androgen deprivation therapy. Proceedings of the Nutrition Society 71. [Crossref]

2. Mohamad H, McNeill G, Haseen F, Ndow J, Craig LCA, et al. (2015) The effect of dietary and exercise interventions on body weight in prostate cancer patients: A systematic review. Nutrition and Cancer 67: 43-60. [Crossref]

3. Kaaks R (2012) Excess body weight, diabetes and cancer: Epidemiologic evidence implicating hormonal and metabolic mechanisms. BMC Proceedings 6.

4. Cohen J (1968) Weighted kappa: nominal scale agreement with provision for scaled disagreement or partial credit. Psychol Bull 70: 213-220. [Crossref]

5. Moher D, Liberati A, Tetzlaff J, Altman DG (2009) Preferred reporting items for systematic reviews and meta-analyses: The PRISMA statement. BMJ (Online) 339: 332-336. [Crossref]

6. Mays N, Pope C, Popay J (2005) Systematically reviewing qualitative and quantitative evidence to inform management and policy-making in the health field. $J$ Health Serv Res Policy 10 Suppl 1: 6-20. [Crossref]

7. Thomas R, Williams M, Bellamy P (2013) A polyphenol rich whole food supplement reduces PSA progression in men with prostate cancer in a double blind placebo controlled RCT-the UK national Pomi-T study. Supportive Care in Cancer 2: S33-S34 [Crossref]

8. Sharma P, Wisniewski A, Braga-Basaria M, Xu X, Yep M, et al. (2009) Lack of an effect of high dose isoflavones in men with prostate cancer undergoing androgen deprivation therapy. J Urol 182: 2265-2272. [Crossref]

9. Caso J, Masko EM, Ii JA, Poulton SH, Dewhirst M, et al. (2013) The effect of carbohydrate restriction on prostate cancer tumor growth in a castrate mouse xenograft model. Prostate 73: 449-454. [Crossref]

10. Westerlund A, Steineck G, Bälter K, Stattin P, Grönberg H, et al. (2011) Dietary supplement use patterns in men with prostate cancer: the Cancer Prostate Sweden study. Ann Oncol 22: 967-972. [Crossref]

11. Keating NL, O’Malley AJ, Freedland SJ, Smith MR (2013) Does comorbidity influence the risk of myocardial infarction or diabetes during androgen-deprivation therapy for prostate cancer? Eur Urol 64: 159-166. [Crossref]

12. Spratt DE, Zhang C, Zumsteg ZS, Pei X, Zhang Z, et al. (2013) Metformin and prostate cancer: Reduced development of castration-resistant disease and prostate cancer mortality. European Urology 63: 709-716. [Crossref]

13. Ziaran S, Goncalves FM, Sn JB (2013) Complex metabolic and skeletal changes in men taking long-term androgen deprivation therapy. Clin Genitourin Cancer 11: 3338. [Crossref]

14. Ansari MS, Gupta NP (2004) Lycopene: a novel drug therapy in hormone refractory metastatic prostate cancer. Urol Oncol 22: 415-420. [Crossref]

15. Vaishampayan U, Hussain M, Banerjee M, Seren S, Sarkar FH, et al. (2007) Lycopene and soy isoflavones in the treatment of prostate cancer. Nutr Cancer 59: 1-7. [Crossref]

16. Lebret T, Coloby P, Descotes JL, Droupy S, Geraud M, et al. (2010) Educational toolkit on diet and exercise: Survey of prostate cancer patients about to receive androgen deprivation therapy. Urology 76: 1434-1439. [Crossref]

17. Goonewardene SS, Persad R, Young A, Makar A (2014) Re: Liam Bourke, Stephen Gilbert, Richard Hooper, et al. Lifestyle changes for improving disease-specific quality of life in sedentary men on long-term androgen-deprivation therapy for advanced prostate cancer: A randomised controlled trial. Eur Urol 66: e51-e52. [Crossref]

18. Smith MR (2008) Androgen deprivation therapy and risk for diabetes and cardiovascular disease in prostate cancer survivors. Curr Urol Rep 9: 197-202. [Crossref]

19. Mostaghel EA, Solomon KR, Pelton K, Freeman MR, Montgomery RB (2012) Impact of circulating cholesterol levels on growth and intratumoral androgen concentration of prostate tumors. PLoS One 7: e30062. [Crossref]

20. Gallagher EJ, LeRoith D (2013) Diabetes, antihyperglycemic medications and cancer risk: smoke or fire? Curr Opin Endocrinol Diabetes Obes 20: 485-494. [Crossref]

21. Dueregger A, Heidegger I, Ofer P, Perktold B, Ramoner R, et al. (2014) The use of dietary supplements to alleviate androgen deprivation therapy side effects during prostate cancer treatment. Nutrients 6: 4491-4519. [Crossref]

22. Grossmann M, Wittert G (2012) Androgens, diabetes and prostate cancer. Endocr Relat Cancer 19: F47-62. [Crossref]

23. Hussain S, Alibhai SMH (2009) ADT's effect on new-onset cardiovascular disease and diabetes. American Journal of Hematology Oncology 8 
24. Philippou Y, Hadjipavlou M, Khan S, Rane A (2013) Complementary and alternative medicine (CAM) in prostate and bladder cancer. BJU Int 112: 1073-1079. [Crossref]
25. Siddique HR, Nanda S, Parray A, Saleem M (2012) Androgen receptor in human health: A potential therapeutic target. Current Drug Targets 13: 1907-1916. [Crossref]

Copyright: (C2015 Goonewardene SS. This is an open-access article distributed under the terms of the Creative Commons Attribution License, which permits unrestricted use, distribution, and reproduction in any medium, provided the original author and source are credited. 